Document downloaded from:

http://hdl.handle.net/10251/121773

This paper must be cited as:

Sekkiou, I.; Chicharro López, FI.; Ortega Tamarit, B.; Mora Almerich, J. (2018). Bidirectional WDM-OOFDM access network based on a sliceable optical transceiver with colorless ONUs. Optical Fiber Technology. 45:98-105. https://doi.org/10.1016/j.yofte.2018.04.015

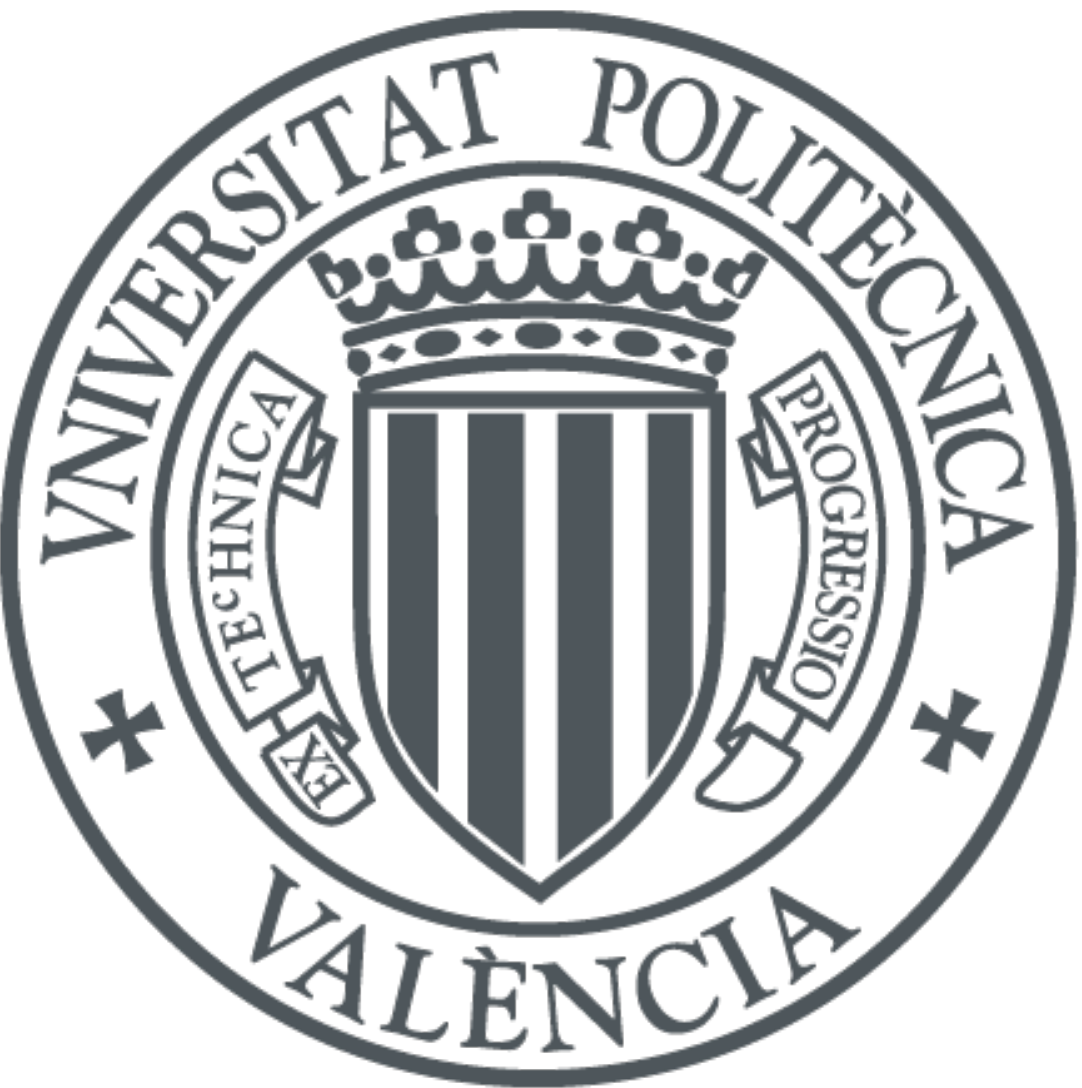

The final publication is available at

http://doi.org/10.1016/j.yofte.2018.04.015

Copyright Elsevier

Additional Information 


\title{
Bidirectional WDM-OOFDM access network based on a sliceable optical transceiver with colorless ONUs
}

\author{
Imene Sekkiou*, Francisco I. Chicharro**, Beatriz Ortega, José Mora \\ ITEAM Research Institute, Universitat Politècnica de València, Valencia, Spain \\ (corresponding author e-mail: bortega@dcom.upv.es)
}

\begin{abstract}
*Permanent address: Elaboration and Characterization Physical, Mechanical and Metallurgical of Materials (ECP3M) Laboratory, Electrical Engineering Department, Faculty of Sciences and Technology, Abdelhamid Ibn Badis University of Mostaganem, 27000, Algeria
\end{abstract}

**Present address: Institute for Multidisciplinary Mathematics, Universitat Politècnica de València, Valencia, Spain

\section{INVITED PAPER}

Abstract-A WDM-OOFDM access network using a centralized optical transceiver based on a single optical broadband sliced source at the Central Office is presented as a low cost solution. Such network offers downlink and uplink signal transmission by using double sideband modulation and optical carrier re-use, as well as dynamic bandwidth allocation, multiple band selection and tunable OFDM band selection in reconfigurable networks at moderate bitrates.

Index Terms - Communications, Optical fiber networks, Access networks, Optical sources, Microwave photonics

\section{INTRODUCTION}

Orthogonal frequency division multiplexing (OFDM) is currently employed as a digital multi-carrier modulation preferred technique over optical carriers in passive optical networks (PON) due to robustness to chromatic dispersion and other impairments as well as its high spectral efficiency and flexible bandwidth allocation [1].

High definition video, multimedia applications or on-line gaming presently drive an exponential growth on high speed broadband and flexible networks deploying data rates of the order of 100 Gbps and beyond. Elastic Optical Networks (EON) have been proposed to improve the wavelength routed optical networks efficiency by offering efficient spectrum management, flexible, scalable and adaptive features [2] as has been also recently implemented in OFDM-PON networks [3].

OFDM access PONs consist of assigning sub-bands of the total signal bandwidth to optical network units (ONUs) in order to implement low-cost ONUs. Optical multiband OFDM systems have been recently proposed for a wide set of applications including dynamic bandwidth allocation in OFDMA schemes showing high granularity and flexible access to a large number of users over tenths of kilometers [4] in addition to the compatibility with both conventional time division multiplexing (TDM) and wavelength division multiplexing (WDM) passive optical networks (PONs) [5].

WDM PON networks allow to support a larger number of ONUs by simply adding wavelengths. However, WDM lasers at each ONU require temperature controllers which drastically increases the overall network cost. A solution to reduce the number of wavelengths is based on colorless WDM PON systems which allow any optical carrier from the Optical Line Terminals (OLT) transporting downstream signals to be re-used for carrying upstream signals [6] and also lead to cost efficient PON architectures [7]. 
Previous works report architectures deploying intensity modulated and directly detected (IM-DD) optical OFDM for downlink (DL) and uplink (UL) for cost effective reasons. Examples of them employ distributed feedback (DFB) lasers [8] and vertical cavity surface emitting lasers (VCSELs) [9] which allowed to demonstrate real-time $11.25 \mathrm{~Gb} / \mathrm{s}$ OOFDM transmission over $25 \mathrm{~km}$ SSMF-based PON systems. End-to-end real time colorless transmissions of IMDD OOFDM signals were also reported by using reflective semiconductor optical amplifiers (RSOA) intensity modulators [10], and furtherly improved REAM-IM-based system allowed to demonstrate bidirectional, real-time, colorless $10 \mathrm{~Gb} / \mathrm{s}$ over $25 \mathrm{~km}$ SSMFs [11]. Other approaches employ RSOAs [12-13] or gain saturated SOAs [14] in the ONU. Moreover, multicarrier sources based on external cavities [15], semiconductor mode-locked [16] or distributed feedback [17] lasers have demonstrated $10 \mathrm{~Gb} / \mathrm{s}$ over $50 \mathrm{~km}$ and beyond by using DDOFDM systems.

However, other approaches in the literature propose to employ DDO-OFDM for DL but deploy a coherent receiver at OLT for the UL signal transmission in order to take advantage of superior performance for UL to enable symmetric data transmission rates. Remotely seeded approaches with separate fibers [17] or employing one sideband for each DL/UL signal transmission [18] are examples to demonstrate symmetrical single-feeder, lightwave centralized OFDMA-PON architectures but a larger number of components is required as the expense for higher performance [19].

Spectrum-slicing of super-luminiscent diodes (SLDs) or optical amplifiers was proposed several years ago [20-21] towards a cost-effective solution for colourless WDM-PONs besides the operating bandwidth limitation induced by the chromatic dispersion and the tradeoff between the loss budget and the sliced bandwidth.

More recently, the utilization of a tunable Mach-Zehnder interferometer (TMZI) allowed to demonstrate an OOFDM access network based on a single optical broadband source (BBS) to transmit moderate bitrates with low cost, high stability, low crosstalk operation [22] and also allowing multiband OFDM transmission in WDM networks [23]. A theoretical analysis in [24] details the potential of this optical BBS-based transmission system over a radio frequency band, also free from carrier suppression effect for any amplitude modulation format. In this paper, we explore the large potentiality offered by the BBS to implement a bidirectional reconfigurable network with dynamic bandwidth allocation, multiple bands operation and multiple users.

The paper is structured as follows: section II describes the proposed network description, section III presents the experimental results on the network performance and finally, section IV summarizes the main conclusions of the paper.

\section{NETWORK DESCRIPTION}

The proposed network employs IM-DD for both the DL and UL, allowing an OLT transceiver at the Central Office ( $\mathrm{CO}$ ) based on a single broadband source which is sliced to generate optical carriers feeding different ONUs, and contains a PIN photodiode (PD) based detector for the UL signal. Simple ONU transceivers include the detection stage for the DL signal detection and an electro-optic modulator (EOM) to remodulate the DL signal with the UL OFDM signal. DL and UL signals employ different frequency bands, which are selected by the proper configuration of both detection stages. The detection stage required for optical OFDM signal transmission using optical broadband sources is based on the use of a tunable Mach-Zehnder interferometer (TMZI) and a PIN photodiode [22], as will be briefly explained below.

Fig. 1 shows the centralized bidirectional OOFDM-WDM architecture and Table 1 includes the experimental details of the required equipment. The optical carrier is generated by a broadband source (BBS) at the OLT (inset (a) shows the output optical spectrum). The broadband optical spectrum is optically sliced by using a multiport tunable optical filter (MTOF) which provides coarse wavelength division multiplexing (CWDM) and allows the selection of the central frequency and the optical bandwidth of the signal supporting each ONU (inset (b) shows the sliced signal carrier assigned to ONU 1).

OLT optical signals are double sideband (DSB) modulated by an OFDM signal in a single-drive electrooptic modulator (EOM) and is multiplexed via a CWDM before transmission over fiber. After transmission, the optical OFDM signals are demultiplexed and, at each ONU, the received signal is split with a passive splitter (PS) where one portion of the signal is detected and the other one is remodulated with the UL signal by means of an EOM.

The electrical multiband OFDM signal is generated offline in Matlab and loaded into an Arbitrary Waveform Generator (AWG), as detailed in the OFDM signal generation block diagram in Fig. 1. An IFFT size of 2048 with QPSK or 16-QAM data-bearing subcarriers satisfying Hermitian symmetry. The 
occupied band is centered at a frequency up to $6 \mathrm{GHz}$, with a 700-1500 MHz -bandwidth in different experiments with a raw data rate below $2 \mathrm{~Gb} / \mathrm{s}$. A cyclic prefix of $6.25 \%$ of the symbol is applied, and equalization in detection process is performed by the inclusion of block-type equispaced pilot pattern of $10 \%$. A pre-emphasis filter is also applied to overcome the non-flat response of the electrical transmitter.

At each ONU, prior to photodetection, there is a TMZI composed by two 50:50 optical couplers and a variable delay line (VDL) in one of the arms. Inset c) in Fig. 1 shows the characteristic TMZI output optical spectrum. As a result of the insertion of this component, the bandwidth limitation arisen from chromatic dispersion in broadband signals is overcome and a bandpass window is generated in the RF transfer function of the overall system. According to the theory [24], the transfer function $H(\Omega)$ corresponding to a rectangular optical carrier spectrum profile can be obtained as the following:

$$
H(\Omega)=\operatorname{sinc}\left(\beta_{2} L \delta \omega_{3 d B} \Omega / 2\right)
$$

where $\beta_{2} L$ is the total fiber chromatic dispersion, $\delta \omega_{3 d B}$ is the $3 \mathrm{~dB}$ optical carrier spectral bandwidth and the new bandpass is centered at the following central frequency $f_{0}\left(\Omega_{0}=2 \pi f_{0}\right)[20]$ :

$$
\Omega_{0}=\Delta \tau / \beta_{2} L,
$$

where $\Delta \tau$ is the differential delay between the MZI fiber arms. Therefore, the transmission bands employed by DL and UL signals will be tuned at their detection stages by proper adjustment of the VDL in the TMZI just before photodetection.

Furthermore, as claimed in [22], this system is free of the power fading penalty due to chromatic dispersion on the spectral region where RF bandpass transmission signal is opened, even when DSB conventional scheme is employed. This fact also simplifies the modulation stage when compared to single-sideband (SSB) modulated signals usually employed to overcome power fading.

Just after the TMZI, the signal is photodetected. The resulting electrical signal is sampled in a real time digital oscilloscope (RTDO). The captured samples are synchronized and parallelized for processing in the regular OFDM receiver blocks. The cyclic prefix is removed, the FFT is applied and the channel is estimated via block-type pilots for equalization purpose. Finally, data are demodulated and the transmitted bit stream is recovered after the serialization stage, as depicted in the OFDM recovery block diagram in Fig. 1.

Table I. Specifications of the required equipment in the OOFDM-WDM proposed network.

\begin{tabular}{|l|l|l|}
\hline & Model & Characteristics \\
\hline AWG & AWG7122C, Tektronix & Sampling rate=24 GS/s \\
\hline BBS & NP Photonics C\&L Band ASE Source & $\begin{array}{l}\text { Optical Power }=19.5 \mathrm{dBm} \\
\text { Wav. Range }=1525-1610 \mathrm{~nm}\end{array}$ \\
\hline MTOF & Finisar WaveShaper 4000S & $\begin{array}{l}\text { Wav.Range }=1527-1568 \mathrm{~nm} \\
\text { Steps of 77 pm } \\
\text { Roll-off value of } 0.06 \mathrm{~dB} / \mathrm{pm}\end{array}$ \\
\hline PS & Fibercore & $\begin{array}{l}\text { V } \pi=5 \mathrm{~V} \\
\text { Insertion loss }(\mathrm{V} \pi)=3.5 \mathrm{~dB}\end{array}$ \\
\hline RTDO & DPO72004C, Tektronix & Insertion losses $=0.5 \mathrm{~dB}$ \\
\hline TMZI & $\begin{array}{l}\text { Fiberco 50:50 couplers } \\
\text { General Photonics Variable Optical } \\
\text { Delay Line (VDL) } \\
\text { Fiberpro polarization controller }\end{array}$ & $\begin{array}{l}\text { Total Insertion Losses }=4 \mathrm{~dB} \\
\text { Max. delay between TMZI arms }=300 \mathrm{ps}\end{array}$ \\
\hline PD & u2t photonics BPDV2020R & $\begin{array}{l}\text { Bandwidth=40 GHz } \\
\text { Responsivity }=0.55 \mathrm{~A} / \mathrm{W}\end{array}$ \\
\hline
\end{tabular}

The remodulated UL signals at different ONUs are launched back to the network through an optical circulator, combined and transmitted over fiber. At the OLT, the received signal is demultiplexed, and a circulator provides de UL signal separation which will be detected after the TMZI stage configured to 
transmit the UL operating band. Inset (d) in Fig. 1 shows the optical spectrum at the output of this MZI, just prior to photodetection. The proposed system allows flexible selection of the OFDM subcarriers band employed for each ONU, both for DL and UL, and also multiband selection is allowed in TMZI multistage configuration, as will be explained below.

The main advantages of our proposed system is the use of a single broadband low cost source to feed multiple ONUs to transmit OFDM signals by IM-DD double sideband modulation for both uplink and downlink, also including carrier reuse for uplink transmission and colorless detection both at OLT and ONU. Optical channels assignment to each ONU and operating OFDM bands can be dynamically changed according to the network conditions and current ONU demands.
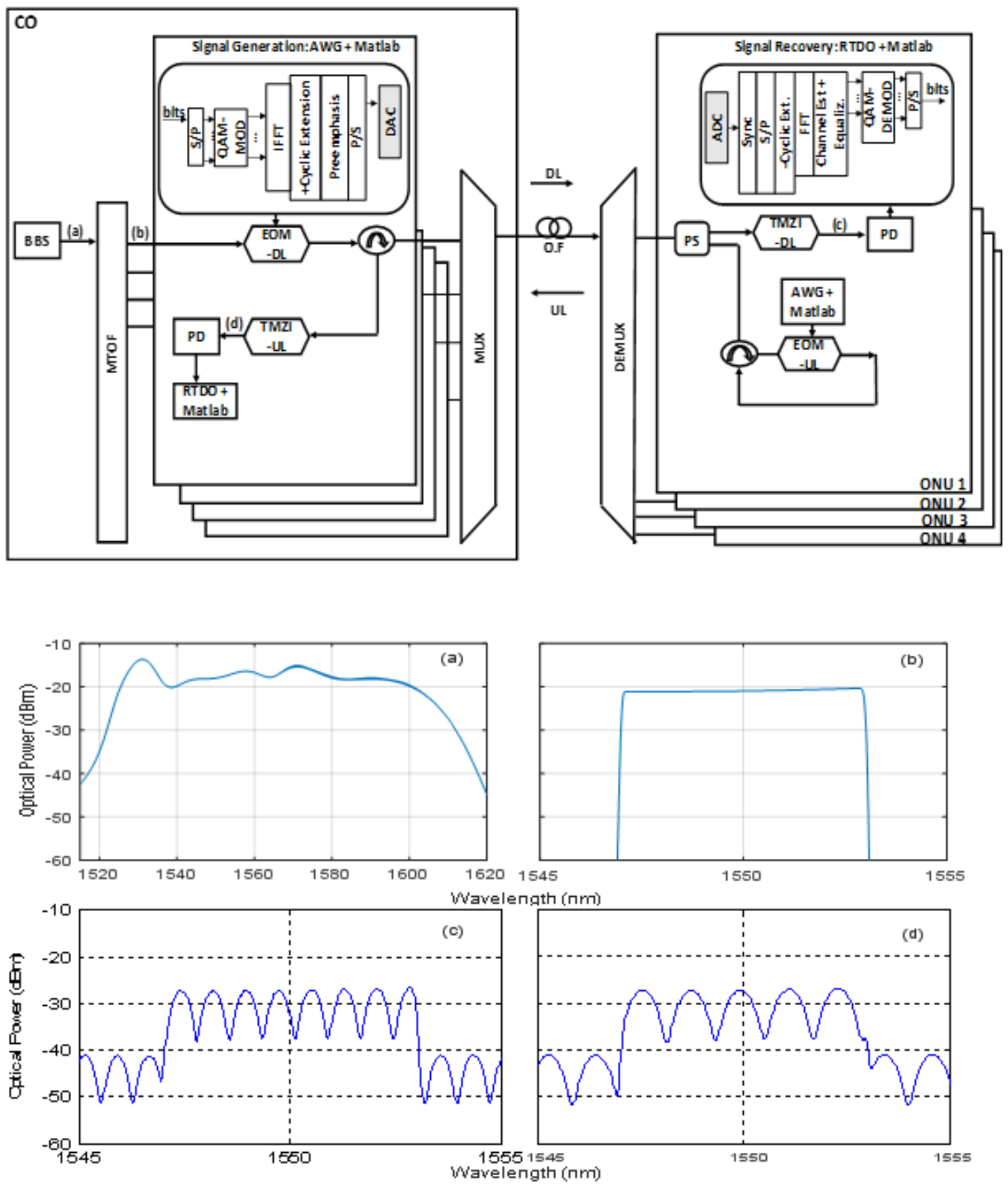

Fig. 1. Centralized bidirectional OOFDM-WDM network. Insets: Optical spectra at different marked positions along the network. BBS: BroadBand Source (Optical), MTOF: Multiport Tunable Optical Filter, EOM: Electro-Optical Modulator, MUX: Multiplexer, DEMUX: Demultiplexer, PS: Passive splitter, TMZI: Tunable Mach-Zehnder Interferometers, DL: Downlink, UL: Uplink, OF: Optical Fiber, PD: Photodetector, AWG: Arbitrary Waveform 
Generator (RF), RTDO: Real Time Digital Oscilloscope, CO: Central Office, ONU: Optical Network Unit. (a): after BBS, (b): after MTOF, (c): after TMZI-DL, (d): after TMZI-UL.

\section{EXPERIMENTAL RESULTS}

Full characterization of the network described above with a $10 \mathrm{~km}$ standard single mode fiber link (dispersion parameter $\left.\beta 2(@ 1550 \mathrm{~nm})=-20 \mathrm{ps}^{2} / \mathrm{km}\right)$ will be done in order to assess its viability as a low cost solution for transmitting optical OFDM signals at moderate bitrates. Flexibility of the proposed network architecture in terms of frequency bands and signal bandwidths for different ONUs will be also shown in a reconfigurable network scenario.

\section{III.a. Carrier re-use for bidirectional transmission}

The sliced optical spectrum corresponding to the signal carrier assigned to ONU 1 is $6 \mathrm{~nm}$ wideband (inset (b) in Fig. 1). The TMZI is configured at this ONU with a differential delay of $\Delta \tau=6.28 \mathrm{ps}$, which, according to eq. (1), leads to a bandpass transmission window centered at $5 \mathrm{GHz}$. The electrical amplitude response of the overall downlink system is measured and depicted in Fig.2 a). It confirms the generation of a new transmission bandpass at $5 \mathrm{GHz}$, with a $3 \mathrm{~dB}$ bandwidth of $0.95 \mathrm{GHz}$, as a result of inserting the TMZI in the ONU, and losses of $10.5 \mathrm{~dB}$ respect to baseband. Full characterization of such transmission system can be found in [22, 24], where the bandwidth of the electrical response is obtained in terms of the optical bandwidth and accumulated dispersion.

The network was tested with a $0.9 \mathrm{~Gb} / \mathrm{s} 16$-QAM OFDM downlink signal with $750 \mathrm{MHz}$ bandwidth. The electrical power spectral density of the recovered DL OFDM signal is also shown in Fig. 2.c), and confirms the effective transmission of the downstream $750 \mathrm{MHz}$ bandwidth OFDM signal band centered at $5 \mathrm{GHz}$.

Fig. 2 b) shows the uplink electrical response for the overall system when the optical carrier, generated at the $\mathrm{CO}$, is reused at the ONU and modulated by an uplink $450 \mathrm{Mb} / \mathrm{s}$ QPSK OFDM signal. In this case, the TMZI at the CO, prior to detection, is configured with a delay $\Delta \tau=4.4 \mathrm{ps}$, which corresponds to a $3.5 \mathrm{GHz}$ transmission band (eq. (1)) with $3 \mathrm{~dB}$ bandwidth of $0.9 \mathrm{GHz}$ and electrical insertion losses of $9 \mathrm{~dB}$. Similarly, the electrical power spectral density of the received UL modulating OFDM signal is also shown in Fig. 2.d), confirming the effective transmission of the upstream $750 \mathrm{MHz}$ bandwidth OFDM signal band centered at $3.5 \mathrm{GHz}$. A residual $5 \mathrm{GHz}$ transmited band at the UL detector can also be observed in Fig. 2.d) where a rejection ratio larger than $14 \mathrm{~dB}$ is obtained.

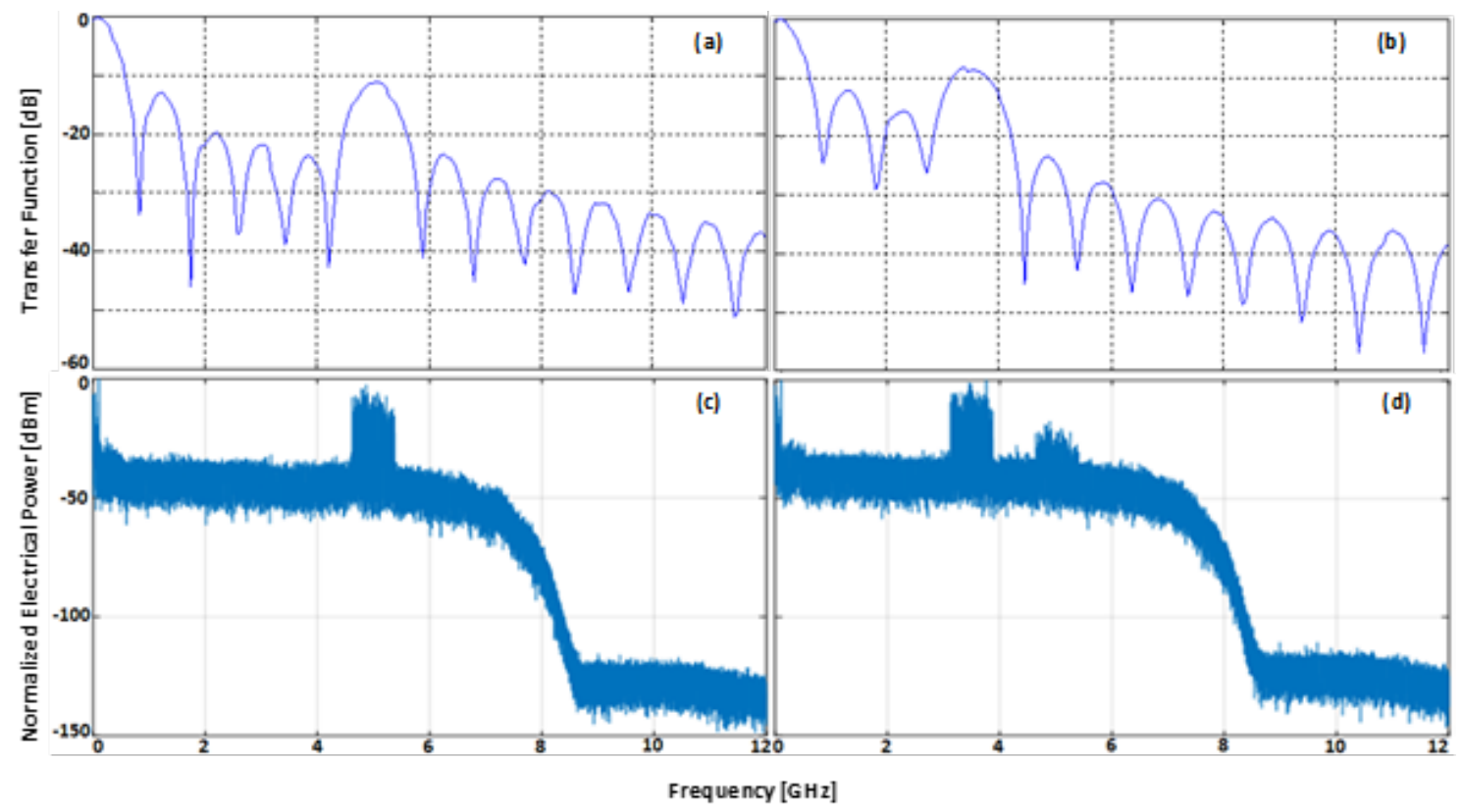

Fig. 2. Electrical transfer function of the proposed system: (a) Downlink, (b) Uplink. Power spectral density of received electrical signal: c) Downstream, (d) Upstream.

Figs. 3 and 4 show the constellation of the electrical back to back (EB2B) and received 16-QAM and QPSK signals corresponding to DL and UL, respectively. EVM was measured for different OFDM 
subcarriers (SC) and also per symbol, with an average $18.97 \%$ EVM value for 16-QAM downlink signal (received optical power $=1.97 \mathrm{dBm}$ ) whereas QPSK uplink signal shows an average EVM per symbol and subcarriers around $13.75 \%$.
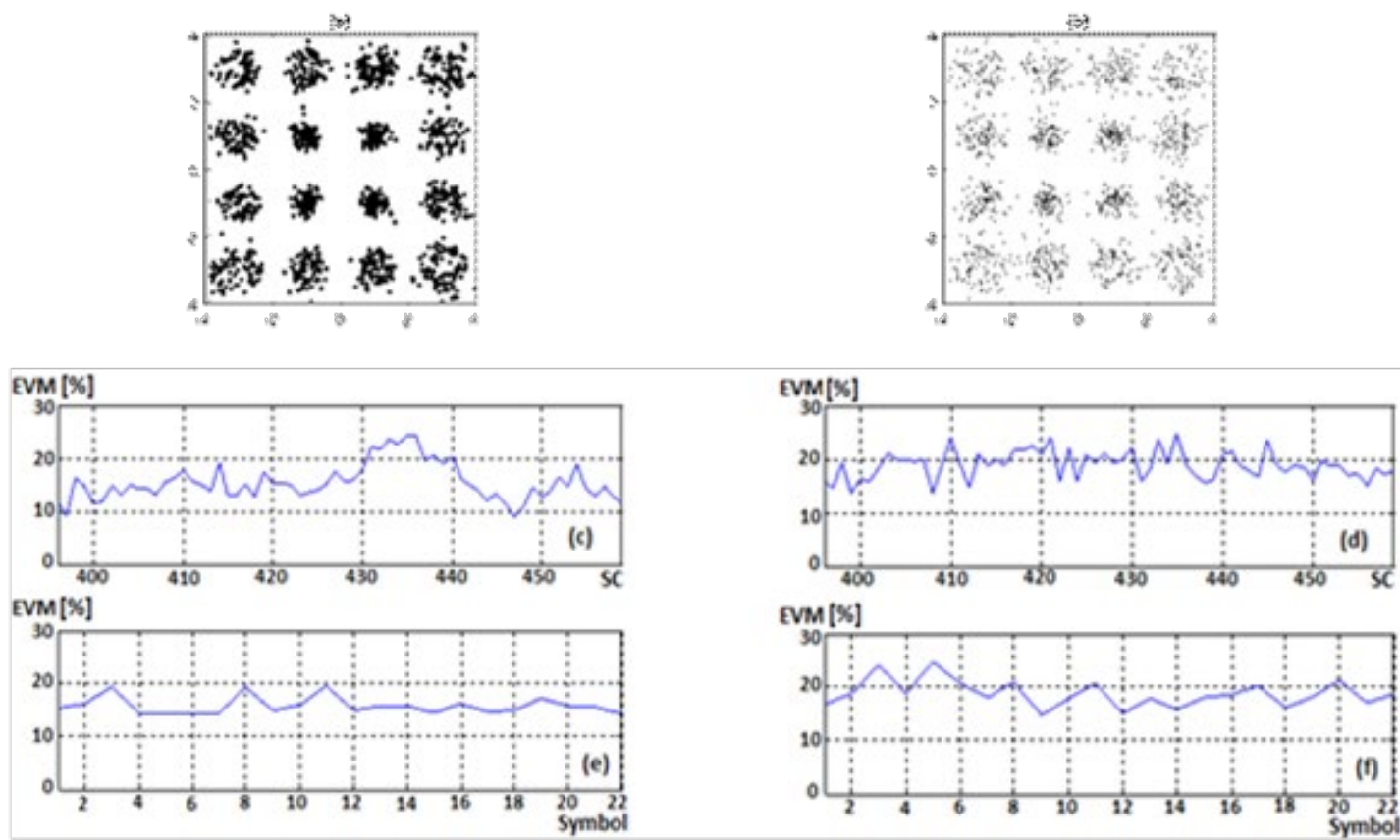

Fig. 3. Downstream transmission: $750 \mathrm{MHz}$ 16-QAM OFDM signal carried at $5 \mathrm{GHz}$. EB2B results: (a) Constellation, (c) EVM per subcarrier, (e) EVM per symbol. After $10 \mathrm{~km}$ fiber transmission link: (b) Constellation, (d) EVM per subcarrier, (f) EVM per symbol.
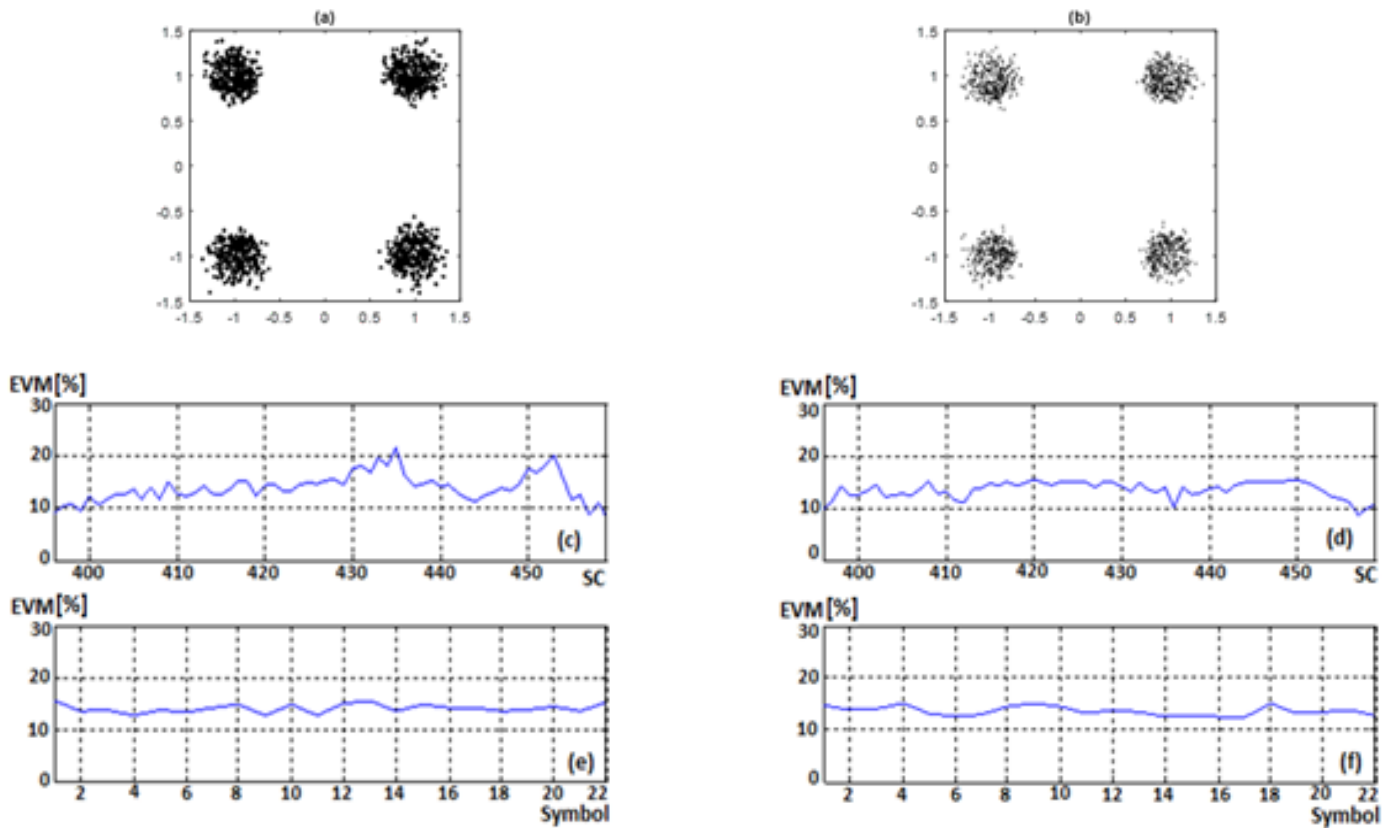

Figure 4. Uplink transmission: $750 \mathrm{MHz}$ QPSK OFDM signal carried at 3.5 GHz. EB2B results: (a) Constellation, (c) EVM per subcarrier, (e) EVM per symbol. After $10 \mathrm{~km}$ fiber transmission uplink: (b) Constellation, (d) EVM per subcarrier, (f) EVM per symbol. 


\section{III.b. Dynamic bandwidth allocation}

The electrical $3 \mathrm{~dB}$ bandwidth of the RF bandpass in our system can be easily obtained in terms of the optical carrier bandwidth and the dispersion characteristic of the link. According to the rectangular profile of the inset (b) in Fig. 1, the bandpass window has a $3 \mathrm{~dB}$ electrical bandwidth $\delta \mathrm{f}_{3 \mathrm{~dB}}$ can be obtained from eq. (1):

$$
\delta \mathrm{f}_{3 \mathrm{~dB}} \sim \frac{0.9 \lambda^{2}}{\left(\beta_{2} L\right) 2 \pi \mathrm{c} \delta \lambda_{3 \mathrm{~dB}}}
$$

where $\delta \lambda_{3 \mathrm{~dB}}$ represents the spectral bandwidth of the optical carrier in the wavelength domain.

Therefore, proper adjustment of the optical bandwidth of the MTOF output port which feeds each ONU determines the transmission bandwidth available at this ONU. In this experiment, a $2.66 \mathrm{GHz}$ 16-QAM OFDM signal centered at $5 \mathrm{GHz}$ was generated as depicts Fig. 5, where the power spectral density of the EB2B as well as its EVM measurement per subcarrier and symbol is plotted. Equipment limitations lead to EVM values close to $20 \%$ when such broadband signal is being generated at the 5 $\mathrm{GHz}$ central frequency.
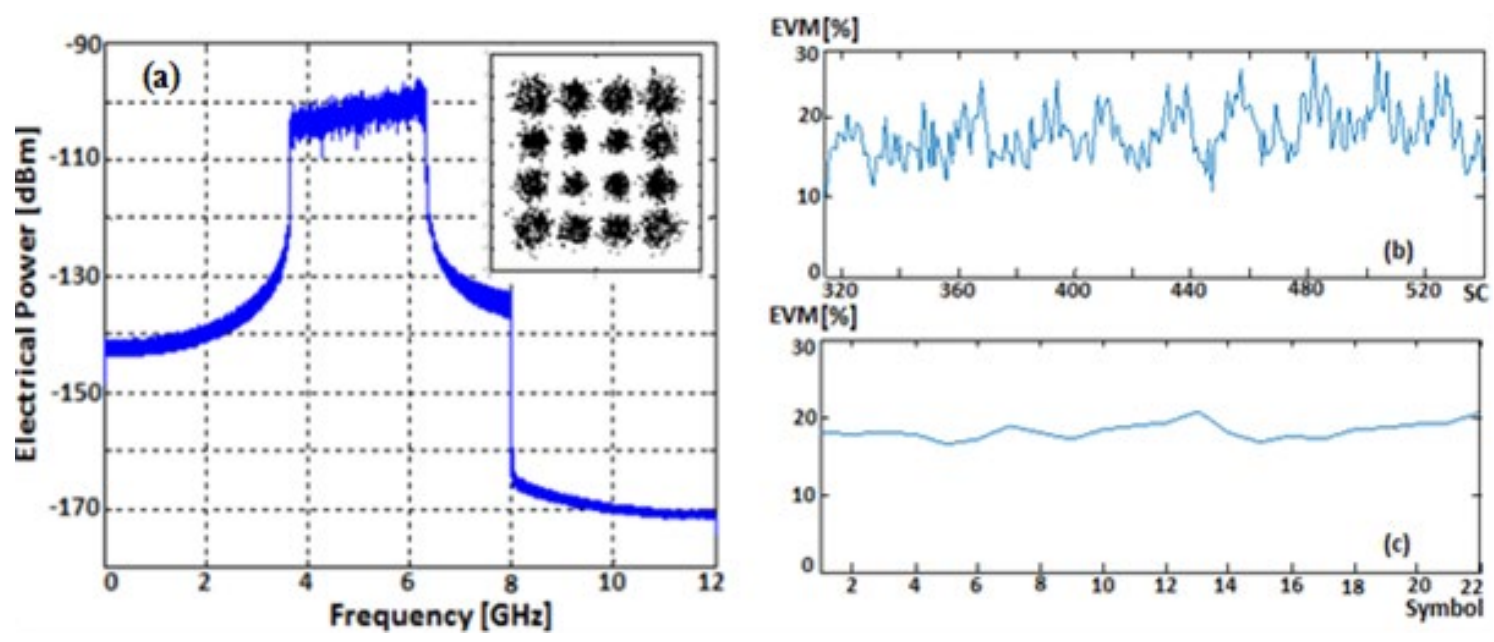

Fig. 5. (a) EB2B power spectral density for BW=2.66 GHz (inset: constellation), (b) EVM per subcarrier, (c) EVM per symbol.

Electrical transfer functions were measured at $5 \mathrm{GHz}$ with different optical bandwidths over a $10 \mathrm{~km}$ fiber link, as shown in Fig. 6 and 7, where the corresponding RF transfer functions have been plotted and also, the electrical spectrum of the recovered signal showing that the effective $3 \mathrm{~dB}$ electrical bandwidth of the transmitted signal is 1.568 and $0.664 \mathrm{GHz}$ when the optical bandwidth is set as 3.9 and $8 \mathrm{~nm}$, respectively. Instes in Figs 6.b) and 7.b) show the relative EVM value respect to the minimum EVM value at the central subcarrier for the sake of an easier comparison. This minimum EVM value obtained at the center of the transmitted band is lower than $35 \%$.

EVM measurements show that the quality of the signal is drastically worse for out of band subcarriers. 


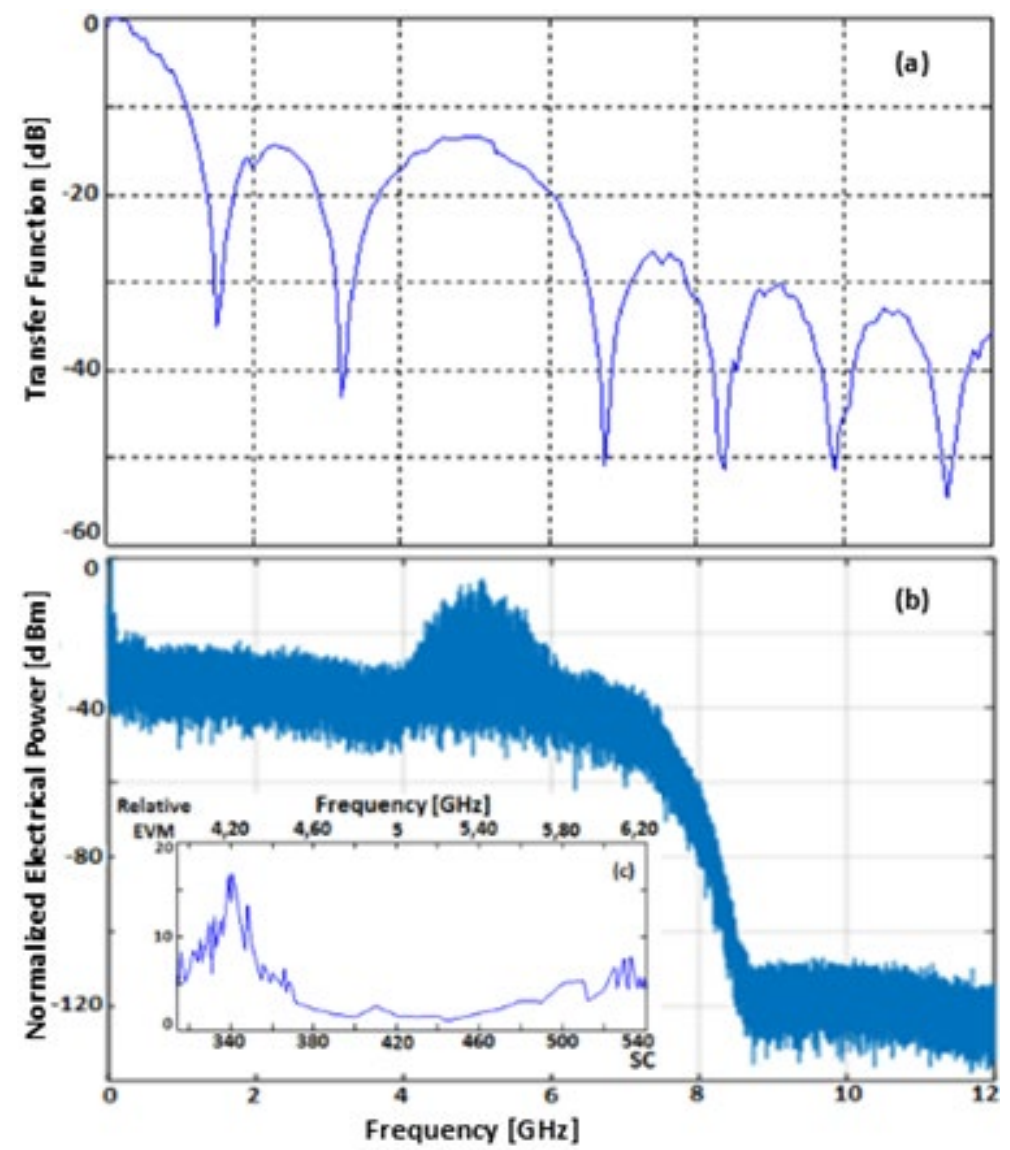

Fig. 6. Transmission system response when optical carrier bandwidth is $3.9 \mathrm{~nm}$ (modulating signal depicted in Fig.5): (a) Electrical transfer function, (b) Power spectral density of recovered electrical signal, (c) EVM per subcarrier. 


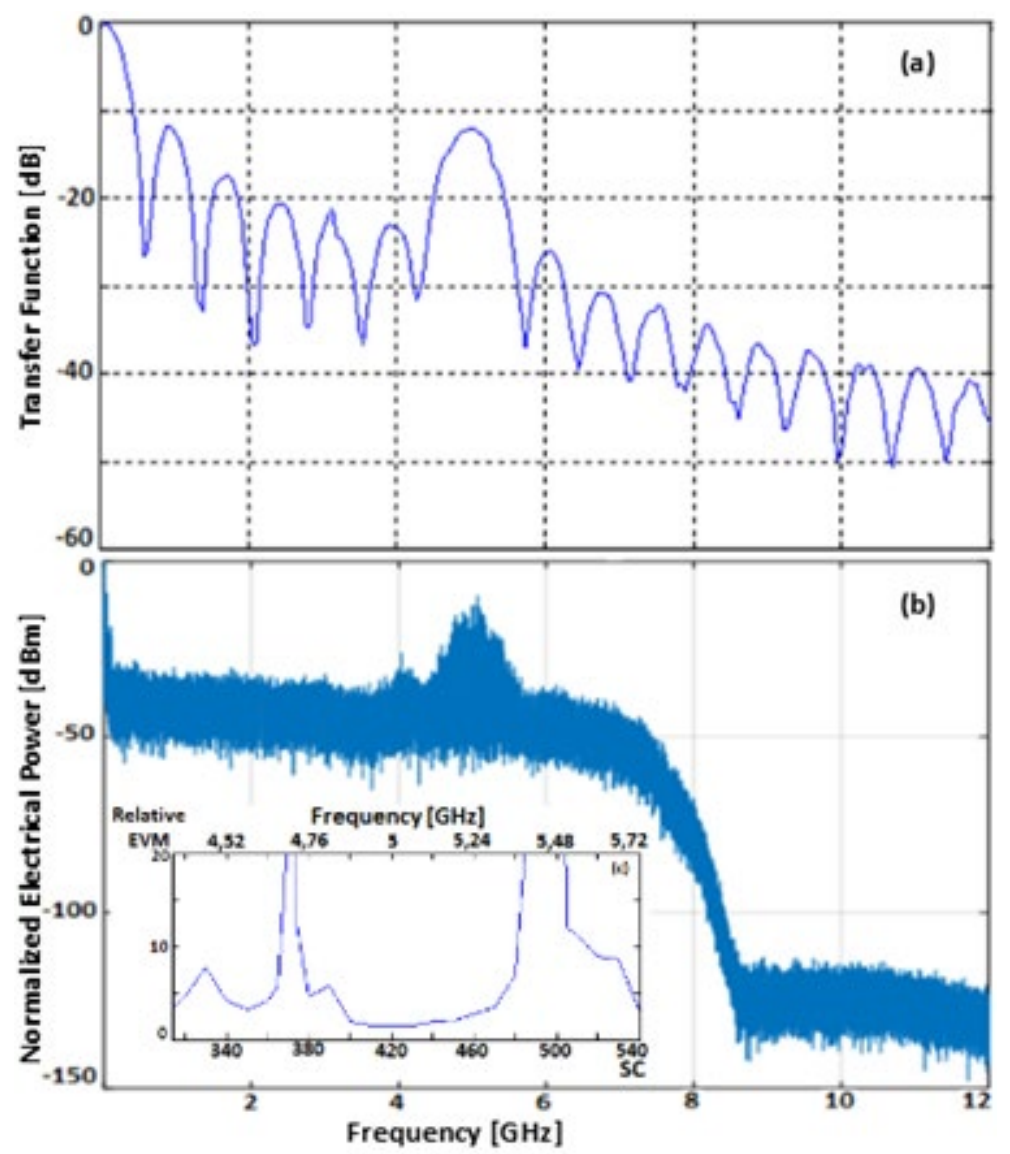

Fig. 7. Transmission system response when optical carrier bandwidth is $8 \mathrm{~nm}$ (modulating signal depicted in Fig. 5): (a) Electrical transfer function, (b) Power spectral density of recovered electrical signal, (c) EVM per subcarrier.

According to the subcarrier frequency spacing, larger bandwidths lead to higher number of subcarriers, and therefore, different allocated bitrate is transmitted, as follows from table 2.

Table 2. Dynamic bandwidth allocation: Effective bitrates for different optical carrier bandwidths.

\begin{tabular}{|l|l|l|l|}
\hline$\delta \lambda_{3 \mathrm{~dB}}(\mathrm{~nm})$ & \multirow{2}{*}{ Bandwidth $(\mathrm{GHz})$} & \multicolumn{2}{|c|}{ Effective bitrate $(\mathrm{Gb} / \mathrm{s})$} \\
\cline { 3 - 4 } & & QPSK & $16-\mathrm{QAM}$ \\
\hline 3.9 & 1.47 & 0.882 & 1.764 \\
\hline 6 & 0.95 & 0.57 & 1.14 \\
\hline 8 & 0.71 & 0.426 & 0.852 \\
\hline 10 & 0.57 & 0.342 & 0.684 \\
\hline 15 & 0.38 & 0.228 & 0.456 \\
\hline
\end{tabular}

Therefore, the proposed network shows moderate bitrates performance but enables bidirectional transmission as well as dynamic bandwidth allocation by using a low cost solution based on a broadband source, as demonstrated above.

\section{III.c. Multiple users in reconfigurable networks}

The proposed scheme in Fig. 1 is valid under reconfigurable networks since the MTOF is a flexible component which dynamically assigns part of the optical spectrum emitted by the broadband optical source and provided the configuration at the TMZI is adjusted according to the dispersion term of the fiber link $\left(\beta_{2} L\right)$ in eq. (2). Fig. 8 shows the spectral bands assigned to four ONUs in the WDM network. The length of the links connecting the CO with different ONUS are 2.8, 5,10 and $21.2 \mathrm{~km}$, respectively and $5 \mathrm{GHz}$ is the central frequency of the OFDM signals transmitted to these ONUs. Fig. 8 shows the measured electrical frequency transfer functions at each ONU where the TMZIs have been configured with differential delays of $1.76,3.14,6.28$ and $13.32 \mathrm{ps}$, respectively. The insets show recovered the 
power spectral density of the recovered signal and demonstrate effective transmission. Any reconfiguration of the network which implies changes in the fiber link or the total amount of dispersion will require TMZI adjustment to fix the working RF band, whereas, similar dispersion condition links are transparent to any reconfiguration of the network.

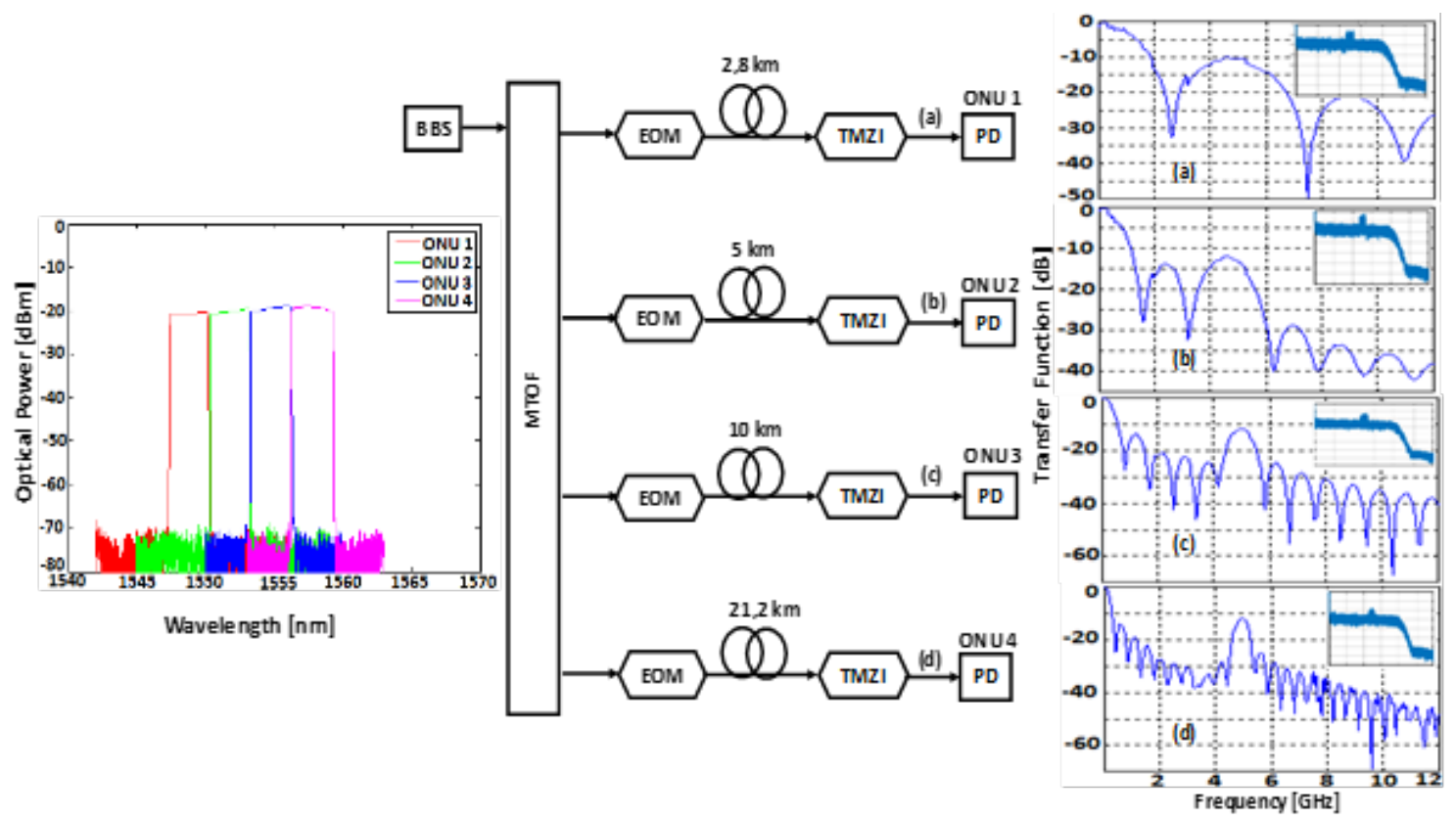

Fig. 8. Optical bands assignment in reconfigurable networks at the $\mathrm{CO}$ output, after the link and after the TMZI) and RF transfer function: a) ONU1 $(2.8 \mathrm{Km})$, b) ONU2 $(5 \mathrm{Km})$, c) ONU3 $(10 \mathrm{Km})$ and d) ONU4 $(21.2 \mathrm{Km})$.

\section{III.d. Multiple band selection}

Finally, we explore the multiple band selection ability of the proposed system. Two TMZI in cascade were configured prior to the downlink detector in order to allow transmission over two different bands. In this case, tunability was also demonstrated in order to provide flexible OFDM multiband assignment to users. Fig. 9 a) shows the experimental setup where both variable delay lines can be independently adjusted before the optical signal photodetection. Figs. 9b) shows the transfer function of the system when each one of the TMZIs is being used (2.5 and $5 \mathrm{GHz}$, respectively) and also the resulting $\mathrm{RF}$ system transfer function when both of them are set in cascade. Two other configurations were set in Figs. $9 \mathrm{c}$ ) and d) leading to the bandpass centered at 3 and $5 \mathrm{GHz}$ and 3 and $6 \mathrm{GHz}$ respectively, but any other set of bands can be achieved. In this case, the quality of the signal was not measured due to 
limitations in the electrical equipment, which power spectral density of broadband electrical signals was not enough to allow proper signal recovery.
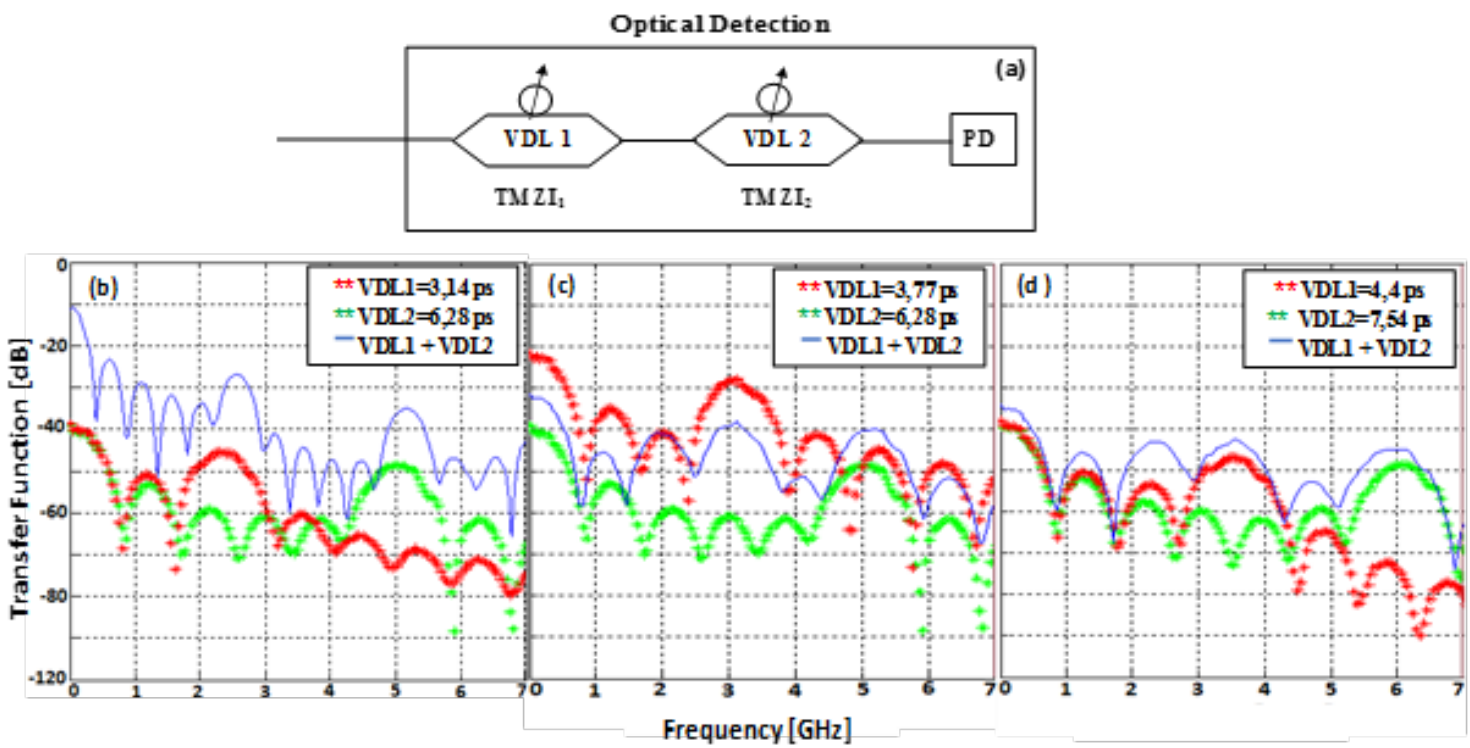

Fig. 9. (a) Experimental detection system with two TMZI in cascade. Electrical RF transfer function: (b) TMZIs set at 2.5 and $5 \mathrm{GHz}$, (c) TMZIs set at 3 and $5 \mathrm{GHz}$, (d) TMZIs set at 3.5 and $6 \mathrm{GHz}$.

\section{CONCLUSION}

This paper presents a low cost WDM-OOFDM network based on a centralized optical broadband source at the Central Office employing double sideband modulation and optical carrier re-use for downlink and uplink transmission below $2 \mathrm{~Gb} / \mathrm{s}$. The flexibility of the approach has been explored to demonstrate dynamic bandwidth allocation, OFDM band selection in reconfigurable networks and multiple tunable OFDM band selection. However, further work is proposed in order to improve the quality of the generated electrical signal to allow signal transmission at higher bitrates.

\section{ACKNOWLEDGMENT}

The authors acknowledge the Research Excellence Award Programme GVA PROMETEO 2017/103 FUTURE MICROWAVE PHOTONIC TECHNOLOGIES AND APPLICATIONS.

\section{REFERENCES}

[1] J. Armstrong, "OFDM for optical communications," Journal of Lightwave Technology, Vol. 27., no. 3, pp. 189204, February 2009.

[2] M. Jinno, H. Takara, B. Kozicki, Y. Tsukishima, Y. Sone, S. Matsuoka, "Spectrum-Efficient and Scalable Elastic Optical Path Network: Architecture, Benefits, and Enabling Technologies", Communications Magazine,Vol.47,no.11, pp. 0163-6804, 2009.

[3] I. N. Cano, X. Escayola, P. C. Schindler, M. C. Santos, V. Polo, J. Leuthold, I. Tomkos, J. Prat, "Experimental Demonstration of a Statistical OFDM-PON With Multiband ONUs and Elastic Bandwidth Allocation [Invited]", Journal of Optical Communications and Networking, Vol. 7., no. 1,pp. A73 - A79, 2015.

[4] T. M. F. Alves, A. V. T. Cartaxo, "Power budget of ultra-dense virtual-carrier-assisted DD MB-OFDM nextgeneration PON," Photonics Technology Letters, Vol. 28., no. 13, pp. 1406-1409, 2016.

[5] K. Kanonakis, I. Tomkos, H. Krimmel, F. Schaich, C. Lange, E. Weis, J. Leuthold, M. Winter, S. Romero, P. Kourtessis, M. Milosavljevic, I. Cano, J. Prat, "An OFDMA-based optical access network architecture exhibiting ultra-high capacity and wireline-wireless convergence," Communications Magazine, Vol. 50., no. 8,pp. $71-78$, 2012.

[6] J. Yu, M. Huang, D. Qian, L. Chen, G. Chang, "Centralized Lightwave WDM-PON Employing 16-QAM Intensity Modulated OFDM Downstream and OOK Modulated Upstream Signals", Photonics Technology Letters, Vol. 20., no. 18,pp. 1545 - 1547,2008.

[7] M. C. Yuang, D. Hsu, P. Tien, H. Chen, C. Wei, S. Chen, J. Chen, "An Energy and Cost Efficient WDM/OFDMA PON System: Design and Demonstration”, Journal of Lightwave Technology, Vol. 31, no. 16, pp. 2809 - 2816, 2013. 
[8] X. Q. Jin, R. P. Giddings, E. Hugues-Salas, J. M. Tang, "Real-time experimental demonstration of optical OFDM symbol synchronization in directly modulated DFB laser-based 25km SMF IMDD systems", Optics Express, Vol. 18., no. 20, pp.21100, 2010.

[9] E. Hugues-Salas, R.P. Giddings, X.Q. Jin, J.L. Wei, X. Zheng, Y. Hong, C. Shu, J.M. Tang, "Real-time experimental demonstration of low-cost VCSEL intensity-modulated 11.95Gb/s optical OFDM signal transmission over 25km PON systems", Optics Express, Vol. 19., no. 4, pp.2979,2011.

[10] X. Q. Jin, J. M. Tang, "Experimental Investigations of Wavelength Spacing and Colorlessness of RSOA-Based ONUs in Real-Time Optical OFDMA PONs",Journal of Lightw. Technol., Vol. 30., no. 16, pp. 2603 - 2609, 2012.

[11] E. Hugues-Salas, R. P. Giddings, X. Q. Jin, Y. Hong, T. Quinlan, S. Walker, J. M Tang, "REAM intensity modulator-enabled $10 \mathrm{~Gb} / \mathrm{s}$ colorless upstream transmission of real-time optical OFDM signals in a single-fiberbased bidirectional PON architecture", Optics Express, Vol. 20., no. 19, pp.21089,2012.

[12] T. Dong, Y. Bao, Y. Ji, A. P. T. Lau, Z. Li, C. Lu, "Bidirectional Hybrid OFDM-WDM-PON System for 40-Gb/s Downlink and 10-Gb/s Uplink Transmission Using RSOA Remodulation”, Photonics Technology Letters, Vol. 24., no. 22, pp. $2024-2026,2012$.

[13] K. Lefebvre, A. T. Nguyen, L. A. Rusch, , "Enabling In-Band Bidirectional OFDM-Uplink and OOK-Downlink Transmission in Long-Reach RSOA- Based WDM-PON Systems", Journal of Lightwave Technology, Vol. 32, no. 20 , pp. 3854 - 3860,2014 .

[14] H. Chen, M. Yuang, P. Tien, D. Hsu, C. Wei, Y. Tsai, J. Chen, "Design and demonstration of a colorless WDMOFDMA PON system architecture achieving symmetric $20 \mathrm{~Gb} / \mathrm{s}$ transmissions with residual interference compensation", Optics Express, Vol. 21., no. 18, pp.21097, 2013.

[15] W.-R. Peng, I. Morita, H. Takahasi, T. Tsuritani, "Transmission of high-speed (>100 Gb/s) direct-detection optical superchannel," Journal of Lightwave Technology, Vol. 30., no. 12, pp. 2025-2034, 2012.

[16] V. Vujicic, P. M. Anandarajah, C. Browning, "WDM-OFDM-PON based on compatible SSB technique using a mode locked comb source," Photonics Technology Letters, Vol. 25., no. 21, pp. 2058-2061, 2013.

[17] N. Cvijetic, M. Cvijetic, M. F. Huang, E. Ip, Y. K. Huang, T. Wang, "Terabit optical access networks based on WDM-OFDMA-PON," Journal of Lightwave Technology, Vol. 30., no. 4, pp. 493-503, 2012.

[18] S. Amiralizadeh, A. T. Nguyen, C. S. Park, L. A. Rusch, "Single-Fiber Lightwave Centralized WDM-OFDMAPON With Colorless Optical Network Units", Journal of Optical Communications and Networking, Vol. 8., no. 4, pp. $196-205,2016$.

[19] P. C. Schindler, R. Schmogrow, M. Dreschmann, J. Meyer, I. Tomkos, J. Prat, H.-G. Krimmel, T. Pfeiffer, P. Kourtessis, A. Ludwig, D. Karnick, D. Hillerkuss, J. Becker, C. Koos, W. Freude, J. Leuthold, "Colorless FDMAPON With Flexible Bandwidth Allocation and Colorless, Low-Speed ONUs [Invited]", Journal of Optical Communications and Networking, Vol. 5., no. 10, pp. A204-A212, 2013.

[20] S. Kaneko, J. Kani, K. Iwatsuki, A. Ohki, M. Sugo, S. Kamei, "Scalability of Spectrum-Sliced DWDM Transmission and Its Expansion Using Forward Error Correction”, Journal of Lightwave Technology, Vol. 24., no. 3, pp. $1295-1301,2006$.

[21] F. Raharimanitra, P. Chanclou, T. N. Duong, J. Le Masson, B. Charbonnier, M. Ouzzif, N. Genay, A. Gharba, F. Saliou, R. Brenot, G. Devalicourt, , "Spectrum Sliced Sources AMOOFDM Modulated for WDM\&TDM PON", European Conference on Optical Communication 2009, , 2009.

[22] F.I Chicharro, B. Ortega, B. De Diego, J. Mora, "OOFDM Signal Transmission Using a Single Optical Broadband Source", Photonics Technology Letters, Vol.29., no:7, pp. 563 - 566, 2017.

[23] F.I Chicharro, B. Ortega, B. De Diego, J. Mora, "Broadband Optical Sources for Low-Cost WDM-MB-OFDM Networks", Communications Letters, Vol.21., no.. 8, pp. 1759 - 1762, 2017.

[24] F.Grassi, J.Mora, B.Ortega, J.Capmany, "Subcarriers multiplexing tolerant dispersion transmission system employing optical broadband sources", Optics Express, Vol. 17., no. 6, pp. 4740-4751, 2009. 